\title{
Performance Evaluation of the Siemens Electronic Portal Imaging Device for IMRT Plan Verification
}

\author{
Y. A. M. Yousif 1,2 , A. J. Van Rensburg1 \\ ${ }^{1}$ Department of Radiation Oncology, Steve Biko Academic Hospital, University of Pretoria, Pretoria, South Africa \\ ${ }^{2}$ Department of Biomedical Physics, Faculty of Health Sciences, Al-Neelain University, Khartoum, Sudan \\ Email: Yousif.medphy@gmail.com, avanrens@medic.up.ac.za
}

Received 4 May 2015; accepted 1 August 2015; published 4 August 2015

Copyright (C) 2015 by authors and Scientific Research Publishing Inc.

This work is licensed under the Creative Commons Attribution International License (CC BY). http://creativecommons.org/licenses/by/4.0/

c) (i) Open Access

\section{Abstract}

A commercially available amorphous Silicon Electronic Portal imaging device (aSi-EPID) installed onto Siemens Oncor Linear accelerator was evaluated with the objective to be implemented for IMRT quality assurance. EPID properties were investigated; these include reproducibility, dependences between the relative EPID responses and field size, dose rate were studied for both photon beams. To determine the inherent build-up of the EPID, varied thickness of Solid-water was placed onto the detector surface and changes in EPID signals were investigated. EPID measurements were compared with ionization chamber measurements (type 30013) connected to a UNIDOS electrometer (PTW-Freiberg) in Water phantom. The use of EPID dose maps was tested by comparing it with TPS-calculated one for IMRT plan applying gamma criteria of $3 \% / 3 \mathrm{~mm}$. The results demonstrated that the aSi-EPID signals were reproducible, and response to the applied MUs were linear up to 100 MUs, and then the response became stable for MUs higher than 200, detector should be calibrated in this range. The results showed a clear dependency on the field size and energy. The dosimetric properties measured in this work shows promise that the aSi-EPID can be used for IMRT verifications.

\section{Keywords}

IMRT, aSi-EPID, Monitor Unit, Field-Size, Dose Rate

\section{Introduction}

Electronic portal imaging devices (EPIDs) have become an important part of modern linear accelerators (Linacs).

How to cite this paper: Yousif, Y.A.M. and Van Rensburg, A.J. (2015) Performance Evaluation of the Siemens Electronic Portal Imaging Device for IMRT Plan Verification. International Journal of Medical Physics, Clinical Engineering and Radiation Oncology, 4, 215-223. http://dx.doi.org/10.4236/ijmpcero.2015.43026 
Previously, EPIDs were introduced to verify patient position. More recently, EPIDs have been employed for dosimetric verification purposes. Another application of EPIDs is for Linac Quality assurance (QA) [1] [2]. The use of EPIDs is based on the conversion of the EPID signal into dose [3]-[7]. The evaluation of the dosimetric properties of EPID is important if EPIDs are to be used for dosimetric purposes. The dosimetric behaviors of the amorphous silicon (a-Si) EPIDs have been studied by several research groups [8]-[12]. However, most of these groups have focused on two commercially available types of the a-Si EPIDs: the Elekta iView GT system and the Varian Portal Vision aS500/1000 [13]-[18]. Today, most modern radiation oncology clinics use Linacs that are equipped with EPIDs that can be used for imaging verification. However, due to the increasing use of IMRT techniques in these clinics, there is a need for efficient methods for QA; EPID dosimetry may serve as an alternative to film dosimetry. Nicolini et al., investigated IMRT dose verifications with an a-Si EPID and found that the EPID can be used for absolute dose measurements [19]. A global calibration model for the use of an a-Si EPID for transit dosimetry has been developed by Nijsten et al. [20]. Studies have shown that the a-Si EPID can be used for the dosimetric verification of dynamic IMRT fields [7] [12]. In general, EPIDs are complicated systems that need to be specially configured for dosimetry purposes, are not independent of the Linac control system that is being tested, and require substantial QC [21]. Although a great deal of work has been done on the aSi EPID dosimetric properties, the interpretation of the EPID characteristics are inconsistent. Additionally, EPID dose responses are still need to be determined despite the fact that many successful and clinical implementation researches have been done on EPID dosimetry. Another reason, the lack of integrated software tools limits the usefulness of a-Si EPID detectors as standard dosimeters for performing QA dosimetric tasks in radiation oncology clinics. However, research efforts to enable EPID dosimetry to serve as an effective component of IMRT QA have been increasing [22]-[26]. Therefore, evaluation of the dosimetric properties of amorphous silicon EPIDs and their applicability for IMRT verification are of current interest [23] [27] [28]. The aim of this work was to evaluate the dosimetric properties of our newly installed a-Si EPID and the feasibility of implementing EPID for IMRT plan verification at Steve Biko Academic Hospital (SBAH).

\section{Materials and Methods}

The EPID and Linear Accelerator: An amorphous silicon (a-Si) EPID (OPTIVUE 1000ART) was used. The flat EPID detector used was a Perkin-Elmer XRD 1640-AG9 detector with an active imaging area of $40 \times 40 \mathrm{~cm}^{2}$ comprising of $1024 \times 1024$ pixels, with a pixel size of $0.8 \mathrm{~mm}$. The panel movements were limited to the vertical direction with varied distances between 115 to $160 \mathrm{~cm}$ source-to-detector distances (SDD). The acquisition software used was Siemens Coherence Therapist Workspace software, version 2.0.09. All measurements were conducted using Siemens ONCOR linear accelerator (Linac) (Siemens Medical solutions, Concord, USA). The Linac equipped with 160 MLC and operates at variable dose-rate mode. Figure 1 shows the geometric set-up of

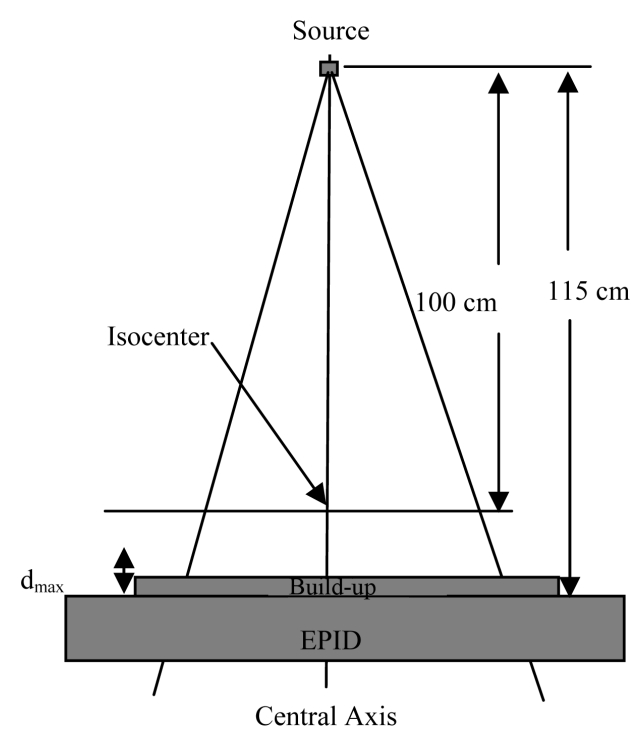

Figure 1. The geometric set-up of the experiment. 
the experiment. For EPID measurements, the relative EPID response (GEPID/Dp) was set as the quotient of the average grayscale value (GEPID) in the region of interest (ROI) of a $10 \times 10$ pixel area and the portal dose (Dp) measured at the same point using ionization chamber for the same set-up. The point-dose measurements were performed in a water phantom using a Farmer ionization chamber (type 30013) connected to a UNIDOS electrometer (PTW, Freiberg, Germany). The 2D measurements were performed EPID. The SDD was varied from 115 to $145 \mathrm{~cm}$ for all of the measurement and to a depth of maximum dose $\left(\mathrm{d}_{\max }\right)$ for the corresponding energies under investigation. 115 was the minimum distance that allowed to position the EPID vertically. This SDD was used because most of the imaging verifications for treatment are conducted at this level. Both the EPID and ionization chamber measurements were conducted on the central axis. All of the ionization chamber measurements were corrected for temperature and pressure variations.

The calibration and reproducibility: The standard calibration of the EPID using a flood-field image with a uniform radiation field and the dark image (non-irradiated image) was performed. This procedure was conducted according to the protocol recommended by the manufacturer and to the calibration guidelines (ONCOR Impression operator's manual) [29]. The short-term reproducibility was evaluated on the basis of 20 subsequent EPID measurements in which $20 \mathrm{MU}$ were delivered using a $10 \times 10 \mathrm{~cm}^{2}$ field size. The long-term reproducibility was evaluated by acquiring 10 consecutive EPID images. The experiment was repeated every two weeks for a period of 3 months.

Monitor Units linearity: Both ionization chamber and EPID measurements were performed sequentially at all times because the EPID and portal doses could not be measured simultaneously. A varying dose was delivered with MUs in the range of 5 to 1000 , using a $10 \times 10 \mathrm{~cm}^{2}$ field size. The low starting values were used because for the application of the EPID for the dosimetry of IMRT fields, low MUs are possible.

Field-size dependence: This was determined for a set of square fields defined by the MLC starting at $1 \times 1$ $\mathrm{cm}^{2}$ and increasing up to $25 \times 25 \mathrm{~cm}^{2}$ without an absorber in the beam. All irradiations were performed by delivering $100 \mathrm{MU}$ at the corresponding dmax for each energy level. Both EPID and ionization chamber sets of measurements were normalized to $10 \times 10 \mathrm{~cm}^{2}$ field-size values.

Dose-rate response: The linearity of the EPID to dose-rate variations was investigated by monitoring the grayscale value on the beam axis for a static field. To modify the dose-rate, the SDD was varied by the distance of the EPID below the isocenter (from 15 up to $60 \mathrm{~cm}$ ). The EPID images were collected each distance, a dose of $100 \mathrm{MU}$ was delivered to a $10 \times 10 \mathrm{~cm}^{2}$ open field.

The effect of the build-up: The response of the EPID as a function of the amount of build-up onto the detector surface was investigated. The EPID measurements were conducted with the outer surface of the EPID. A field of $10 \times 10 \mathrm{~cm}^{2}$ at the isocenter was projected at the extended SDD to give a field of $14.5 \times 14.5 \mathrm{~cm}^{2}$ and 100 MUs were delivered, using the maximum available nominal dose-rate. The build-up material used was the solid-water phantom (GAMMEX RMI, Middleton, WI). A series of measurements were obtained with depths ranging from $0.1 \mathrm{~cm}$ to $4.0 \mathrm{~cm}$ on the EPID. Comparative measurements were performed using ionization chamber for the same set-up.

Clinical Application: The relative dosimetry performance of the EPID for IMRT QA was tested by comparing the EPID dose maps with the calculated dose maps using CMS XiO Treatment Planning System (TPS) for an IMRT plan that contained 7 IMRT fields. The experimental set-up for the EPID and the TPS calculation is shown in Figure 2. Both EPID and TPS dose maps were imported for analysis and evaluation, which were performed by using Radiological Imaging Technology (RIT) 113 Dosimetry Software, Version 5.2 (Colorado Springs, CO) [30]. The software introduced a new calibration method called plan-based calibration, which can be used to develop relative dosimetric calibration curves between the acquired EPID images and the TPS-calculated. It also allows a relative dose comparison to be conducted without conducting a separate calibration. These routines provide a suite of measurements and dose map comparisons including gamma method using gamma criteria of $3 \%$ and $3 \mathrm{~mm}$ (dose difference and distance-to-agreement).

\section{Results}

Reproducibility: The EPID short-term reproducibility characteristics were similar for both beam qualities, as shown in Figure 3(a). For the same number of MUs applied, average grayscale values did not vary significantly and were approximately $0.99 \%$ and $0.97 \%$, and the standard deviation were $0.2 \%$ and $0.3 \%$ for 6 and $15 \mathrm{MV}$, respectively. Figure 3(b) shows the long-term reproducibility over a period of 3 months was found to be within 


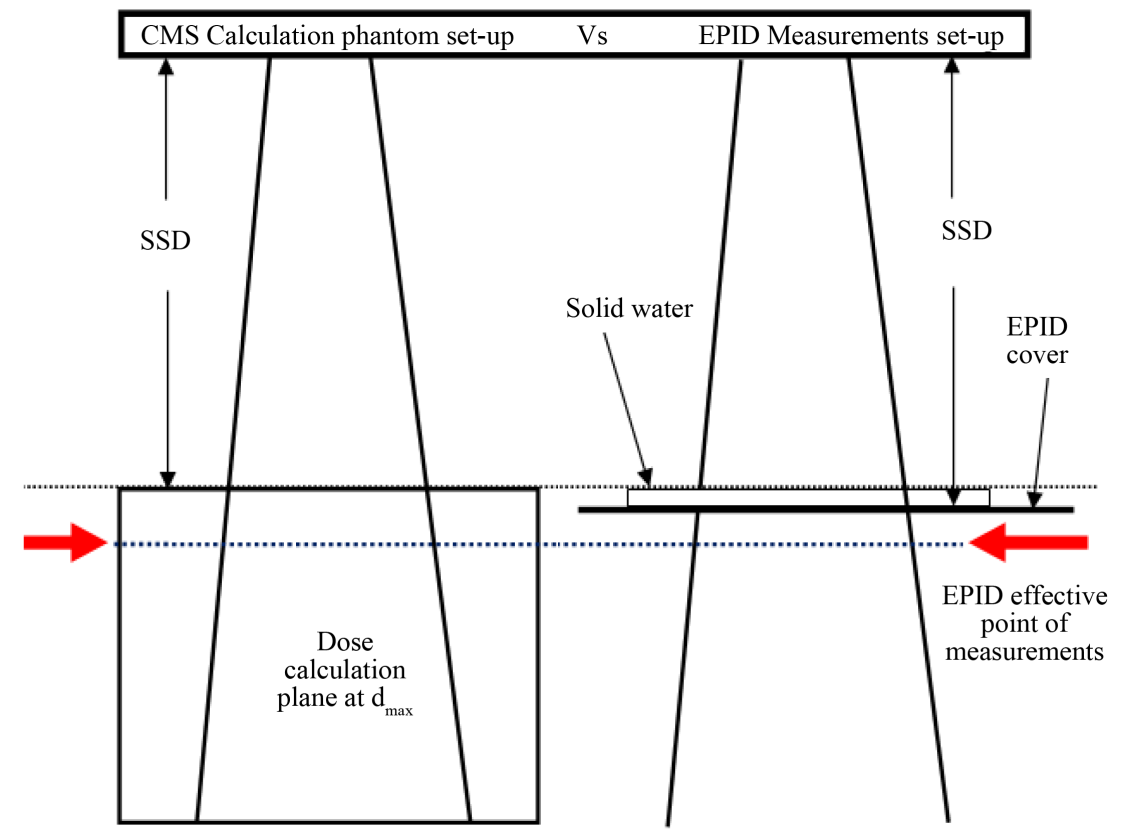

Figure 2. The experimental set-up of the position of the Electronic Portal Imaging device and CMS Treatment planning system (TPS) calculation phantom.

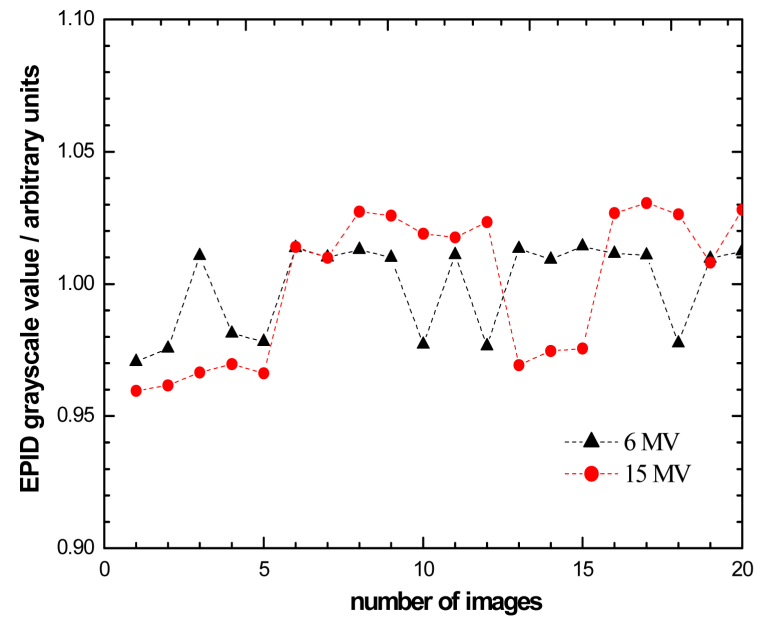

(a)

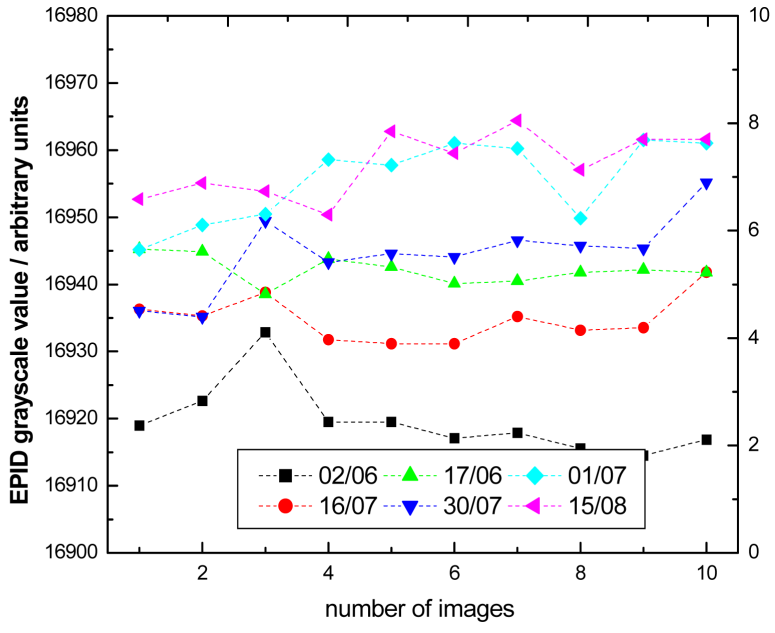

(b)

Figure 3. (a) The variation in the average grayscale values for 20 consecutive measurements of short-term reproducibility; (b) The variations in grayscale values for 10 consecutive measurements of long-term reproducibility over a 3-month period.

$\pm 1.7 \%$ for the $6 \mathrm{MV}$ photon beam.

Monitor Units linearity: The relationship between the EPID response and the applied MUs for a range of 5 to 1000 MUs is shown in Figure 4. The data were fitted in a semi-log graph and the results showed almost identical behavior for both beam qualities. Additionally, the EPID response decreased in the region of low doses for both energies, and this was more obvious for the $15 \mathrm{MV}$. The a-Si EPID response was higher by 2.1, for $6 \mathrm{MV}$ photons and 2.8\% for $15 \mathrm{MV}$ photons for $1000 \mathrm{MUs}$, whereas for the doses at $10 \mathrm{MU}$, the reduction was $4.8 \%$ and $5.8 \%$ for the 6 and $15 \mathrm{MV}$ photons, respectively.

Field-size dependence: Figure 5 shows the results of the relative EPID response with the field size, which, indicates both under-response and over-response areas for both beams. For small field sizes, the EPID response shows less response dependence on the field size up to the $10 \times 10 \mathrm{~cm}^{2}$ field; as the field size increases, the 


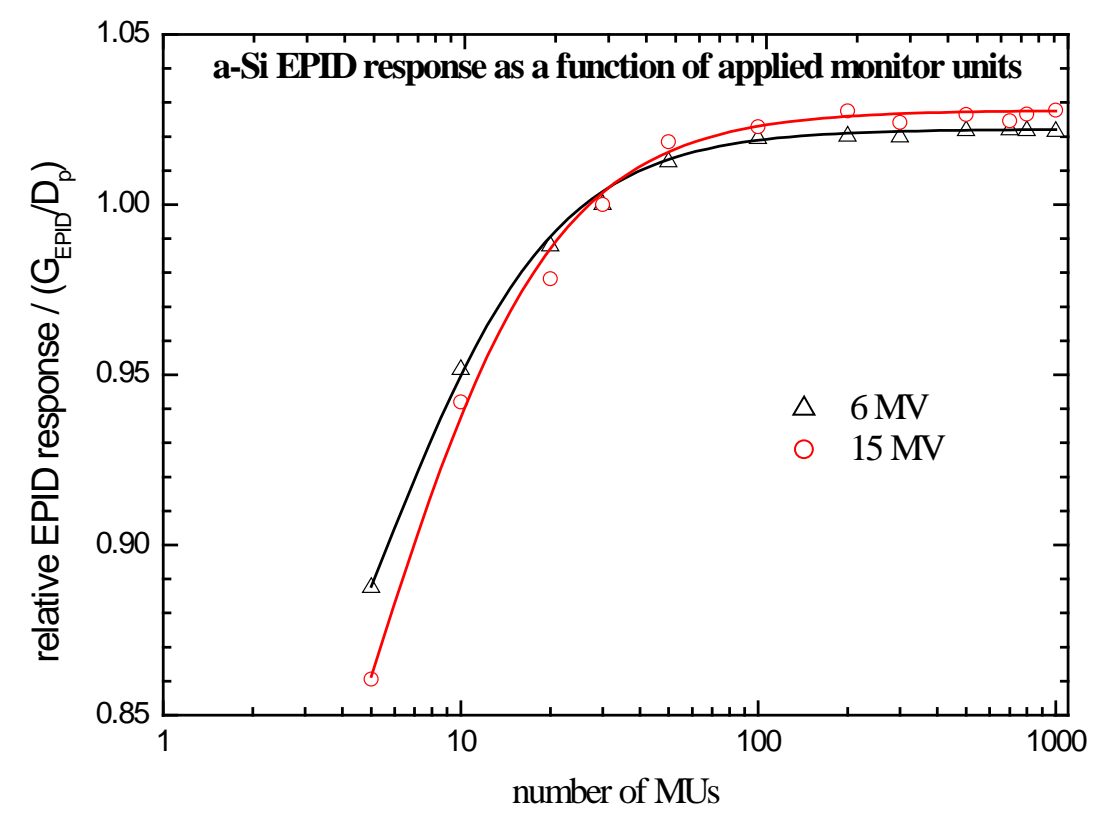

Figure 4. The a-Si EPID response as a function of applied monitor units (MU) for 6 and $15 \mathrm{MV}$ photons.

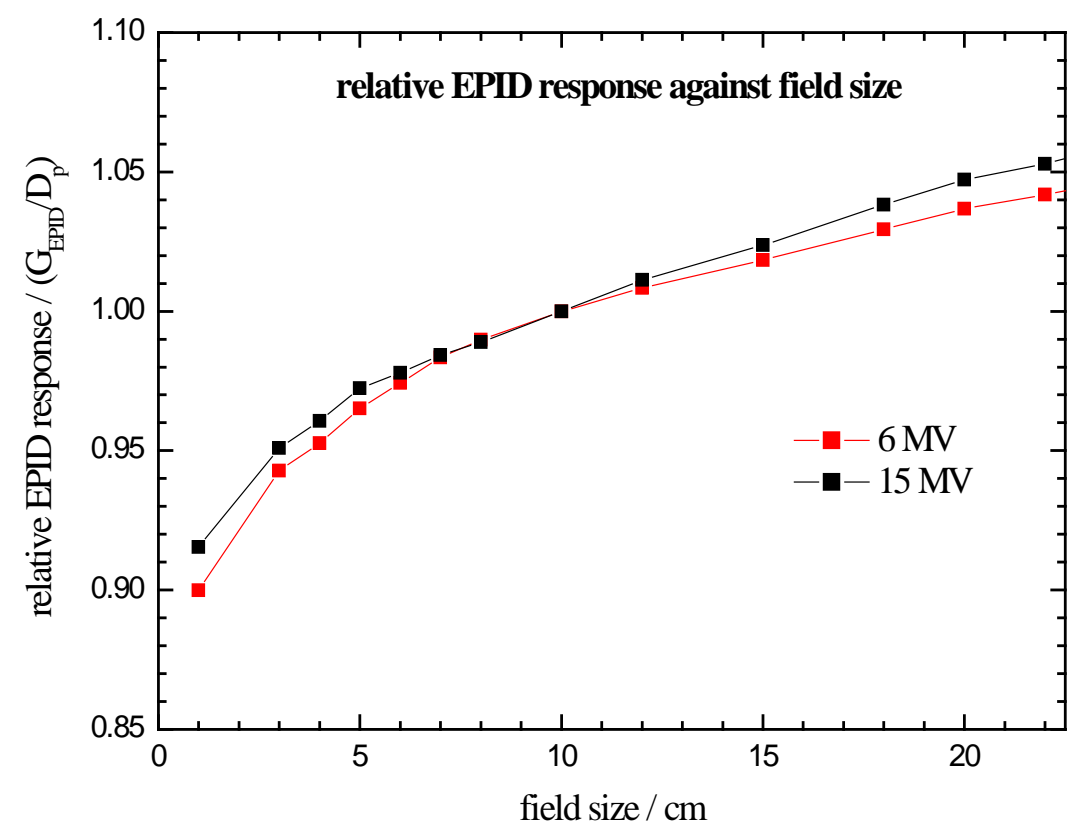

Figure 5. The relative response of the EPID (the ratio of the grayscale value, GEPID, divided by the portal dose, $D_{p}$ as a function of the field size for the 6 and 15 MV photons. Results were normalized for a $10 \times 10 \mathrm{~cm}^{2}$ field.

EPID response shows a clear dependency on the field size and energy. This effect is probably created by changes in scatter with increased field size. Scatter has a low energy component; probably due to presence of high atomic number component in the phosphor material [8]-[10]. The pattern is the same for the two energies with an under-response for small fields and an over-response for the bigger field ranges.

Dose-rate linearity: Figure 6 shows a plot data and a linear function was fit to the data. The linear regression coefficients are $\mathrm{R}^{2}=0.999$ for all the fits. The linear fits also indicated proportionality constants showing that 
the detector is proportional over the entire measured range and does not deviate from the inverse square behavior.

The EPID build-up effect: Figure 7(a) and Figure 7(b) show a plot of EPID signal response versus the depth. As expected, the signal from EPID and ion chamber increases as a build-up material increases up to a depth of a maximum signal. After that, the signal and dose received decreases with depth. Ion chamber measurements are recognized to be proportional to the dose received; accordingly, the depth at which the maximum ion chamber signal occurs will be the depth at which the maximum dose is delivered to the material. Since EPID has some inherent build-up, the maximum signal will be reached with less extra build-up materials added on top. The difference between the two depths of the maximum signal can be considered the amount of water-equivalent material that the inherent build-up of the EPID is contributing. The inherent build-up on EPID was found to be $0.40 \pm$ $0.06 \mathrm{~cm}$ for $6 \mathrm{MV}$ and $0.46 \pm 0.01 \mathrm{~cm}$ for $15 \mathrm{MV}$. This build-up must be taken into account when adding a solid-water phantom material on top to achieve the depth of the maximum dose of the detector. The results will be useful for EPID calibration for dosimetric purposes.

Clinical Application: The relative dose performance of IMRT fields was assessed based on a seven beam, 6

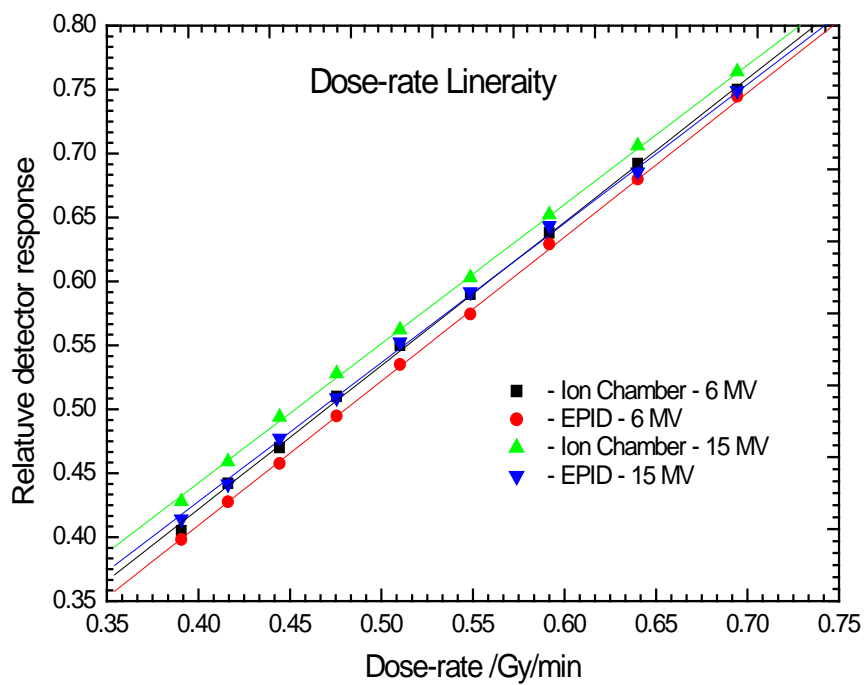

Figure 6. The variation of the dose values as a function of the source-to-detector distance (SDD) measured using the ion chamber and the a-Si EPID for the $6 \mathrm{MV}$ and $15 \mathrm{MV}$ photons.

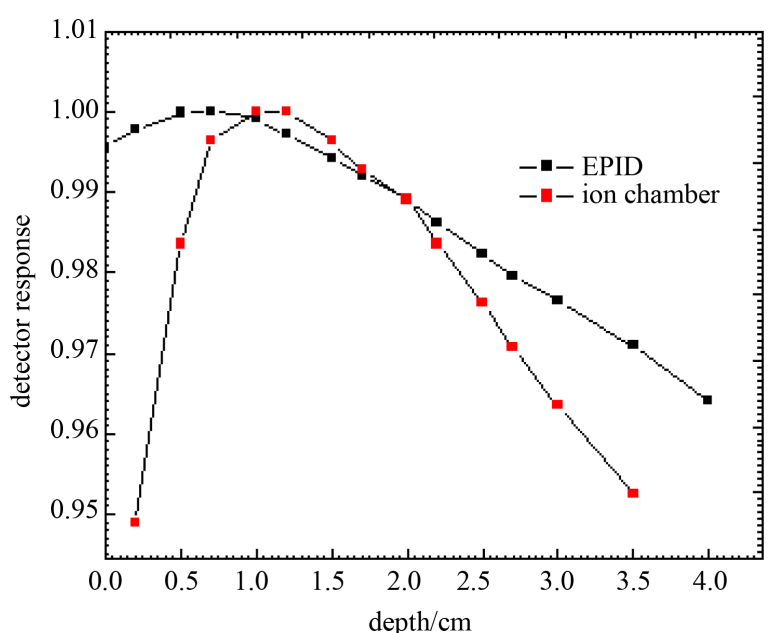

(a)

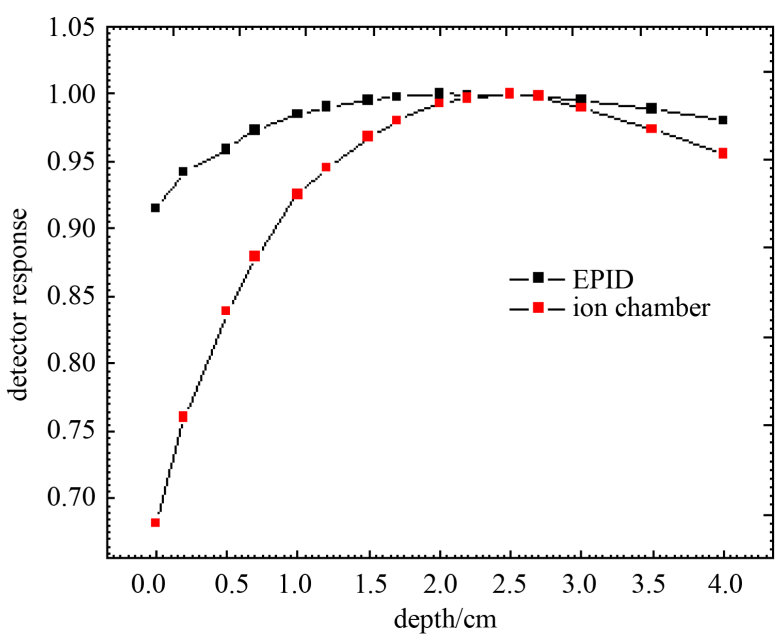

(b)

Figure 7. Present the grayscale values measured by EPID versus the water-equivalent depth $(\mathrm{cm})$ for 6 and 15 MV photons. The depth was placed on the top of the EPID. 
MV head and neck IMRT plans. The agreement between the dose maps measured with EPID and TPS-calculated dose maps were evaluated using 3\%/3mm pass rate criteria. Figure 8 shows and example of dose maps comparisons. The corresponding gamma map shows that most of the field has gamma values of less than or equal to 1.0 (indicated by the large area shown in gray); the small area in the center of the field has gamma values that are greater than 1.0, corresponding to a high dose-gradient region (shown in red). In addition, there are areas at the edges of the field (shown in red) that show gamma values greater than 1.0; most of these areas fell outside the treatment field. Because the majority of the field has gamma values less than or equal to 1.0, and areas with gamma values greater than 1.0 are confined to a high dose-gradient region, there is agreement between the measured and TPS-calculated dose maps.

\section{Discussion and Conclusion}

In this work, the EPID dosimetric performance was investigated for the purpose to be implemented for IMRT plan verification at SBAH. Our results showed that the EPID grayscale values were reproducible. The EPID response to the applied MUs increased linearly up to about 100 MUs, and then it became stable for MUs higher than 200. Therefore, the detector should be calibrated in this range. These results are in agreement with [16] [31].

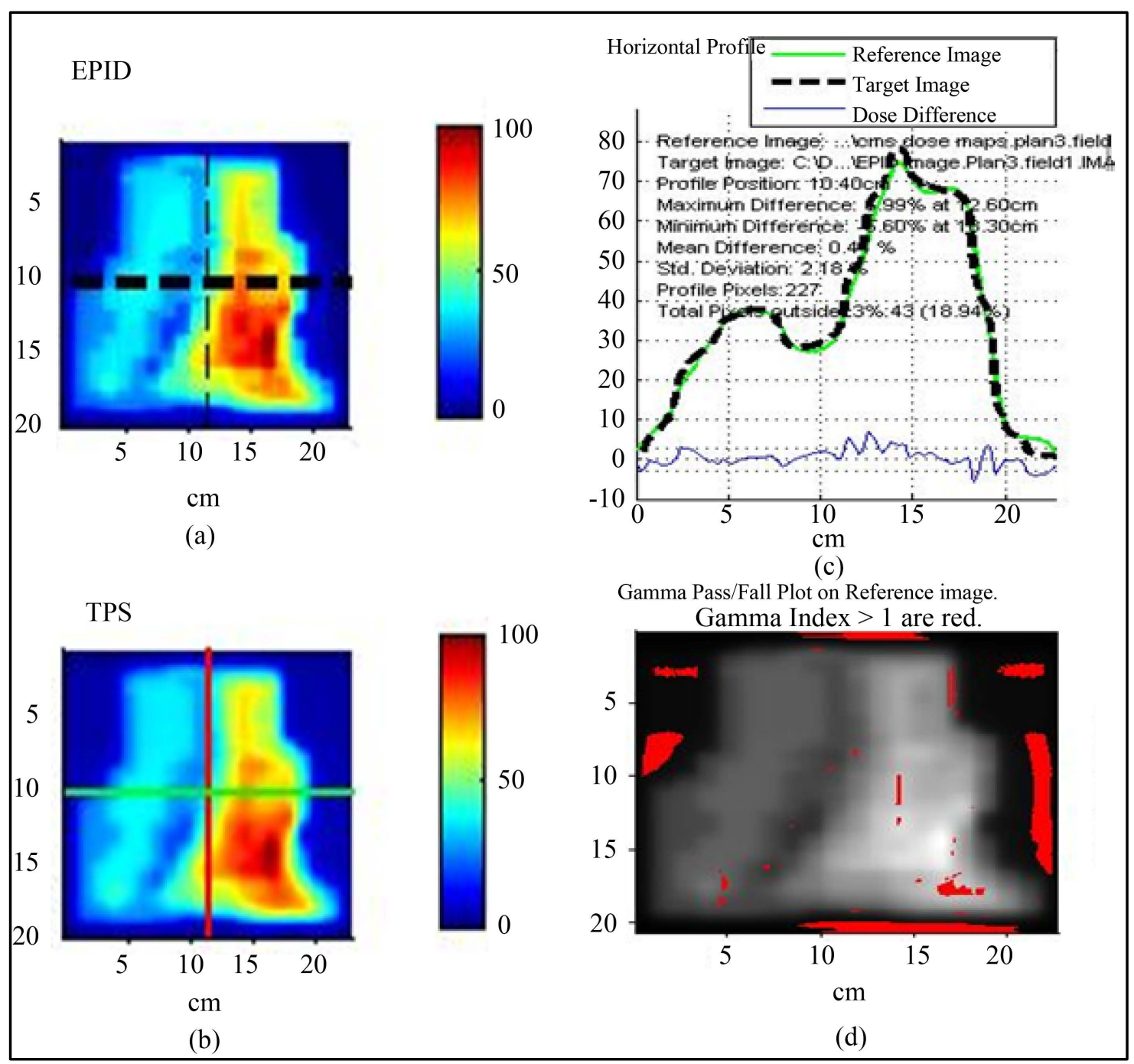

Figure 8. Plan based calibration for EPID. (a) Measured fluence map for IMRT field, (b) TPS calculated fluence map for IMRT field, (c) Dose profiles measured and calculated along the lines of the a-Si EPID images and the TPS images, and (d) A gamma index was $\leq 1$ for the DTA and the $\triangle$ Dmax criteria of $3 \mathrm{~mm}$ and $3 \%$. 
The EPID response shows a clear dependency on the field size and energy. The EPID response was also in close agreement with other studies for different field sizes. Greer et al. measured an under-response of $2.0 \%$ for a $4 \times$ $4 \mathrm{~cm}^{2}$ field and 2.5\% for a $24 \times 24 \mathrm{~cm}^{2}$ field, but did not correct for EPID off-axis response [8]. Van Esch et al. examined the response for varying field sizes at two different energies and their approach also depended on the SDD [10]. The EPID showed a liner response to the dose-rate with a proportional coefficient of $\mathrm{R}^{2}=0.999$ for both photon beams. The dose-rate linearity of the EPID has been previously reported [10]. Thus, only verification was performed in this work to establish that the EPID at SBAH performs as expected. The inherent build-up of EPID was determined by comparing the measurements with the ion chamber. The results will be useful for the EPID calibration for dosimetric purposes. The results demonstrate that the plan based calibration method is suitable for use with EPID images to perform. The experimental set-up used to validate the calculated dose maps was highly satisfactory. In general, the a-Si EPID showed the prerequisite dosimetric characteristics for performing the Patient-Specific IMRT QA.

\section{References}

[1] Nicolini, G., Vanetti, E., Clivio, A., Fogliata, A., Boka, G. and Cozzi, L. (2006) Testing the Portal Imager GLA as Algorithm for Machine Quality Assurance. Radiation Oncology, 3, 1-20.

[2] Greer, P.B. and Barnes, M.P. (2007) Investigation of an Amorphous Silicon EPID for Measurement and Quality Assurance of Enhanced Dynamic Wedge. Physics in Medicine and Biology, 52, 1075-1087. http://dx.doi.org/10.1088/0031-9155/52/4/014

[3] Heijmen, B.J., Pasma, K.L., Kroonwijk, M. and Altohof, V.G.M. (1995) Portal Dose Measurement in Radiotherapy Using an Electronic Portal Imaging Device (EPID). Physics in Medicine and Biology, 40, 1943-1955. http://dx.doi.org/10.1088/0031-9155/40/11/012

[4] Chen, J., Chuang, C.F., Morin, O., Aubin, M. and Pouliot, J. (2006) Calibration of an Amorphous-Silicon Flat Panel Portal Imager for Exit-Beam Dosimetry. Medical Physics, 33, 3095-3105. http://dx.doi.org/10.1118/1.2168294

[5] Partridge, M., Hesse, B.-M. and Muller, L. (2002) A Performance Comparison of Direct-and Indirect-Detection Flat Panel Imager. Nuclear Instruments and Methods in Physics Research, 48, 351-363. http://dx.doi.org/10.1016/S0168-9002(01)02023-X

[6] Chang, J., Mageras, G.S., Chui, C.S., Ling, C.C. and Lutz, W. (2000) Relative Profile and Dose Verification of Intensity-Modulated Radiation Therapy. International Journal of Radiation Oncology * Biology * Physics, 47, 231-240. http://dx.doi.org/10.1016/S0360-3016(99)00555-6

[7] Pasma, K.L., Kroonwijk, M., de Boer, J.C.J., Visser, A.G. and Heijmen, B.J. (1998) Accurate Portal Dose Measurement with a Fluoroscopic Electronic Portal Imaging Device (EPID) for Open and Wedged Beams and Dynamic Multileaf Collimation. Physics in Medicine and Biology, 43, 2047-2060. http://dx.doi.org/10.1088/0031-9155/43/8/004

[8] Greer, P. and Popescu, C. (2003) Dosimetric Properties of an Amorphous Silicon Electronic Portal Imaging Device for Verification of Dynamic Intensity Modulated Radiation Therapy. Medical Physics, 30, 1618-1627. http://dx.doi.org/10.1118/1.1582469

[9] Grein, E.E., Lee, R. and Luchka, K. (2002) An Investigation of a New Amorphous Silicon Electronic Portal Imaging Device for Transit Dosimetry. Medical Physics, 29, 2262-2628. http://dx.doi.org/10.1118/1.1508108

[10] Van Esch, A., Depuydt, T. and Huyskens, D.P. (2004) The Use of an aSi-Based EPID for Routine Absolute Dosimetric Pre-Treatment Verification of Dynamic IMRT Fields. Radiotherapy and Oncology, 71, 223-234. http://dx.doi.org/10.1016/j.radonc.2004.02.018

[11] Podesta, M., Nijsten, S.M., Snaith, J., Orlandini, M., Lustberg, T., Emans, D., et al. (2012) Measured vs. Simulated Portal Images for Low MU Fields on Three Accelerator Types: Possible Consequences for 2D Portal Dosimetry. Medical Physics, 39, 7470-7479. http://dx.doi.org/10.1118/1.4761950

[12] Warkentin, B., Rathee, S. and Steciw, S. (2012) 2D Lag and Signal Nonlinearity Correction in an Amorphous Silicon EPID and Their Impact on Pretreatment Dosimetric Verification. Medical Physics, 39, 6597-6608. http://dx.doi.org/10.1118/1.4757582

[13] Louwe, R.J., Tielenburg, R., van Ingen, K.M., Mijneer, B.J. and van Herk, M.B. (2004) The Stability of Liquid-Filled Matrix Ionization Chamber Electronic Portal Imaging Devices for Dosimetry Purposes. Medical Physics, 31, 819-827. http://dx.doi.org/10.1118/1.1668411

[14] Antonuk, L.E., El-Mohri, Y. and Huang, W. (1998) Initial Performance Evaluation of an Indirect-Detection, Active Matrix Flat-Panel Imager (AMFPI) Prototype for Megavoltage Imaging. International Journal of Radiation Oncology*Biology*Physics, 42, 437-454. http://dx.doi.org/10.1016/S0360-3016(98)00210-7

[15] El-Mohri, Y., Antonuk, L.E., Yorkston, J., Jee, K.-W., Maolinbay, M., Lam, K.L. and Siewerdsen, J.H. (1999) Relative 
Dosimetry Using Active Matrix Flat-Panel Imager (AMFPI) Technology. Medical Physics, 26, 1530-1541. http://dx.doi.org/10.1118/1.598649

[16] Winkler, P., Hefner, A. and Georg, D. (2005) Dose-Response Characteristics of an Amorphous Silicon EPID. Medical Physics, 32, 3095-3105. http://dx.doi.org/10.1118/1.2040711

[17] McDermott, L.N., Nijsten, S.M., Sonke, J.J., Partridge, M., Herk, M. and Mijnheer, B.J. (2006) Comparison of Ghosting Effects for Three Commercial a-Si Electronic Portalimaging Devices. Medical Physics, 33, 2448-2451. http://dx.doi.org/10.1118/1.2207318

[18] McDermott, N.L., Louwe, R.J., Sonke, J.J., van Herk, M. and Mijnheer, B.J. (2004) Dose-Response and Ghosting Effects of an Amorphous Silicon Electronic Portal Imaging Device. Medical Physics, 31, 285-295. http://dx.doi.org/10.1118/1.1637969

[19] Nicolini, G., Fogliata, A., Vanetti, E., Clivio, A. and Vetterli, D. (2006) GLAaS: An Absolute Dose Calibration Algorithm for an Amorphous Silicon Portal Imager. Applications to IMRT Verifications. Medical Physics, 33, $2839-2851$. http://dx.doi.org/10.1118/1.2218314

[20] Nijsten, S.M.J.J., Van Elmpt, W.J.C., Jacobs, M., Mijnheer, B.J., Dekker, A.L.A.J., Lambin, P. and Minken, A.W.H. (2007) A Global Calibration Model for a-Si EPIDs Used for Transit Dosimetry. Medical Physics, 34, 2872-2884. http://dx.doi.org/10.1118/1.2776244

[21] Van Elmpt, W., Ezzell, G.A. and Orton, C.G. (2009) EPID Dosimetry Must Soon Become an Essential Component of IMRT Quality Assurance. Medical Physics, 36, 4325-4327. http://dx.doi.org/10.1118/1.3213082

[22] Nijsten, S.M., Mijnheer, B.J., Dekker, A.L., Lambin, P. and Minken, A.W. (2007) Routine Individualized Patient Dosimetry Using Electronic Portal Imaging Devices. Radiotherapy and Oncology, 83, 65-75. http://dx.doi.org/10.1016/j.radonc.2007.03.003

[23] Van Elmpt, W., McDermott, L.N., Nijsten, S., Wendling, M., Lambin, P. and Mijnheer, B.J. (2008) A Literature Review of Electronic Portal Imaging for Radiotherapy Dosimetry. Radiotherapy and Oncology, 88, 289-309. http://dx.doi.org/10.1016/j.radonc.2008.07.008

[24] van Elmpt, W., Nijsten, S., Mijnheer, B.J., Dekker, A. and Lamb, A. (2008) The Next Step in Patient-Specific QA: 3D Dose Verification of Conformal and Intensity-Modulated RT Based on EPID Dosimetry and Monte Carlo Dose Calculations. Radiotherapy and Oncology, 86, 86-92. http://dx.doi.org/10.1016/j.radonc.2007.11.007

[25] Cilla, S., Fidanzio, A., Greco, F., Sabatino, D., Russo, A., Gargiulo, L., Azario, L. and Piermattei, A. (2010) Correlation Functions for Elekta aSi EPIDs Used as Transit Dosimeter for Open Fields. Journal of Applied Clinical Medical Physics, 12, 218-233.

[26] Deshpande, S., Vial, P. and Holloway, L. (2011) 2-D Radiation Therapy Dosimetry Using EPIDs: Dose Response Variation between 3 Siemens Electronic Portal Imaging Devices (EPIDs). Radiation Measurements, 46, 1916-1919. http://dx.doi.org/10.1016/j.radmeas.2011.08.006

[27] Deshpande, S., Holloway, L., Metcalfe, P. and Vial, P. (2012) An Integrated 2-D Dosimeter and Electronic Portal Imaging Device for in Vivo Dosimetry: A Feasibility Study. International Journal of Radiation Oncology*Biology* Physics, 84, S764. http://dx.doi.org/10.1016/j.ijrobp.2012.07.2044

[28] Van Esch, A., Huyskens, D.P., Hirschi, L., Scheib, S. and Baltesn, C. (2013) Optimized Varian aSi Portal Dosimetry: Development of Datasets for Collective Use. Journal of Applied Clinical Medical Physics, 14, 4286.

[29] ONCOR Impression Operator Manual, Siemens Medical Solutions USA, Inc., Malvern, PA.

[30] Radiological Imaging Technology (RIT). Colorado Springs, CO. http://www.radimage.com/

[31] Greer, P.B. (2007) Off-Axis Dose Response Characteristics of an a-Si Electronic Portal Imaging Device. Medical Physics, 34, 3815-3824. http://dx.doi.org/10.1118/1.2779944 